\title{
The effect of animacy on metamemory
}

\author{
Ping $\mathrm{Li}^{1} \cdot \mathrm{Xiaoyu} \mathrm{Jia}^{2} \cdot \mathrm{Xinyu} \mathrm{Li}^{1} \cdot$ Weijian $\mathrm{Li}^{1}$
}

Published online: 18 February 2016

(C) Psychonomic Society, Inc. 2016

\begin{abstract}
Previous research has shown that the animacy quality of materials affects basic cognitive processes such as memory (i.e., animate stimuli are remembered better than are inanimate stimuli). This is referred to as the animacy effect. Little research has examined, however, whether this effect can be extended to higher cognitive processes such as metamemory. In the present studies, we investigated the influence of animacy on judgments of learning (JOLs) and the underlying basis of the animacy effect, namely, processing fluency and beliefs about the animacy effect. In Experiment 1, participants studied animate and inanimate words and made immediate JOLs. Results revealed that participants gave higher estimates for animate than they did for inanimate words. In Experiments $2 a$ and $2 b$, we evaluated the contribution of processing fluency to the animacy effect either by measuring self-paced study time or by disrupting fluency by presenting half of the words in an easy or difficult font style. Results from both experiments indicated that processing fluency contributes minimally to the animacy effect. In questionnaire-based Experiment 3, participants estimated hypothetical participants would better
\end{abstract}

Xinyu Li

xyli@zjnu.cn

Weijian Li

xlxh@zjnu.cn

Ping Li

liping_psychology@163.com

Xiaoyu Jia

jiaxiaoyu@126.com

1 Department of Psychology, Zhejiang Normal University, Yingbin Road 688, Jinhua 321004, Zhejiang Province, China

2 Department of Psychology and Behavioural Sciences, Zhejiang University, Hangzhou, China remember the animate words than the inanimate words, suggesting the potential role of beliefs on the animacy effect on JOLs. To conclude, these findings suggest that animacy is a reliable cue when people monitor their learning in higher cognitive processes. The beliefs, not processing fluency, contribute substantially to the animacy effect on JOLs.

Keywords Metamemory $\cdot$ Judgments of learning $\cdot$ Animacy · Fluency $\cdot$ Beliefs

Several lines of evidence suggest that the animacy quality of materials plays an important role in visual, attentional, and linguistic processing (Caramazza \& Mahon, 2003; New, Cosmides, \& Tooby, 2007; Pratt, Radulescu, Guo, \& Abrams, 2010; Szewczyk \& Schriefers, 2011; but see Lowder \& Gordon, 2015, for an alternative explanation). Recent research by Nairne, VanArsdall, Pandeirada, Cogdill, and LeBreton (2013) has demonstrated that animacy is also a critical mnemonic dimension, and animate words are remembered better than are inanimate words. This is referred to as the animacy effect (see also Bonin, Gelin, \& Bugaiska, 2014; VanArsdall, Nairne, Pandeirada, \& Blunt, 2013; VanArsdall, Nairne, Pandeirada, \& Cogdill, 2014). This finding is in line with the functionalist view of memory put forward by Nairne and his colleagues (Nairne \& Pandeirada, 2008, 2010; Nairne, Pandeirada, Gregory, \& VanArsdall, 2009; Nairne, Pandeirada, \& Thompson, 2008), which states that the human memory system has been tuned to solve critical adaptive problems. For example, Nairne, Thompson, and Pandeirada (2007) found that encoding words in a survival condition, such as rating common nouns for their survival relevance (e.g., in securing food, water, or protection from predators), as compared to several control conditions (e.g., rating words according to pleasantness), led to better long-term retention. 
The findings of several recent studies suggest that the higher order cognitive processes, such as metamemory (e.g., judgments of learning [JOLs] and study-time allocation), also could have a relevant functional role (Li et al., 2015; Metcalfe \& Jacobs, 2010; Palmore, Garcia, Bacon, Johnson, \& Kelemen, 2012). For instance, Metcalfe and Jacobs (2010) argued that people try to optimize their study-time allocation in a learning environment, similar to other species that try to optimize their efficiency in foraging situations. Humans' success as learners depends on the effectiveness of these strategies, just as animals' evolutionary fitness depends on their foraging effectiveness. In one of our previous studies, we also found that activating a mate-search or mate-guarding motive led the male participants to allocate more time to highly attractive female faces than to less attractive ones, suggesting the adaptive significance of self-regulated learning ( $\mathrm{Li}$ et al., 2015).

Given that animacy plays a role in the human memory system, it may also affect metamemory, which is considered a higher order cognitive process. However, as the animacy dimension has been studied only very recently in the memory literature, no research has investigated the effect of animacy on metamemory (i.e., memory monitoring in this study). This was the first focus of the current study. Specifically, we sought to examine whether the animacy quality of materials influences learners' memory monitoring during encoding (i.e., predictions about the likelihood of remembering recently studied information, assessed via JOLs).

Second, we sought to determine how animacy affects learners' JOLs. The effect of animacy on JOLs could potentially be mediated by two factors: processing fluency and beliefs about memory (e.g., Koriat, Bjork, Sheffer, \& Bar, 2004; Mueller, Dunlosky, \& Tauber, 2015; Mueller, Dunlosky, Tauber, \& Rhodes, 2014; Mueller, Tauber, \& Dunlosky, 2013). Concerning the former, processing fluency has been identified as an important basis for JOLs in a number of previous studies, with more fluent processing leading to higher JOLs (Castel, McCabe, \& Roediger, 2007; Hertzog, Dunlosky, Robinson, \& Kidder, 2003; Koriat, 2008; Koriat \& Ma'ayan, 2005; Undorf \& Erdfelder, 2011, 2015). For instance, Besken and Mulligan (2013) found that participants gave higher JOLs for intact words than they did for backwardmasked words. It was suggested that greater perceptual fluency during encoding is taken as an indicator of future memorability. In the present case, perhaps the ease with which animate or inanimate items are processed directly influences people's JOLs. For example, Bonin et al. (2014) had participants engage in an animate-inanimate categorization task. Animate words were categorized reliably faster than were inanimate words, suggesting that animate items are easier to process than are inanimate items, and such processing fluency, in turn, could lead to higher JOLs for animate than for inanimate items.
With respect to beliefs, previous studies have found that beliefs contribute substantially to the generation (Begg, Vinski, Frankovich, \& Holgate, 1991; Matvey, Dunlosky, \& Guttentag, 2001), relatedness (Koriat et al., 2004; Mueller et al., 2015; Mueller et al., 2013), font size (Kornell, Rhodes, Castel, \& Tauber, 2011; Mueller et al., 2014), word frequency (Jia et al., 2016), and physical weight (Alban \& Kelley, 2013) effect on JOLs. For example, Alban and Kelley found that the weight effect on JOLs was eliminated by explicitly varying the meaning of weight through the priming manipulation (i.e., lightweight is good, heavyweight is bad), suggesting that people may have a belief about the perceptual experiences of weight (i.e., weight signifies importance) and use this belief to make JOLs. Animacy of items may influence JOLs because people have a belief about how this cue affects memory, and it is this belief that mediates the animacy effect on JOLs. For example, people may have an underlying belief that animate stimuli are more important than are inanimate stimuli, as they would be more important for our human ancestors as potentially dangerous entities in need of quick identification (VanArsdall et al., 2014). This belief may be generated prior to the experiment and then activated from long-term memory when a JOL is made (Koriat et al., 2004). Alternatively, it may be developed when people are asked to make JOLs for animate and inanimate words. As suggested by Mueller et al. (2015), when participants are explicitly instructed to make a JOL, they may adopt an analytic problem-solving mode (analytic-processing theory; AP) to reduce uncertainty in their predictions. For these reasons, people may give animate items higher JOLs than inanimate items.

In sum, the current study investigated the role of animacy in memory monitoring. Using Chinese animate and inanimate words, our main goal was to examine whether animacy influences JOLs (i.e., participants would give higher JOLs to animate words compared to inanimate words). If this is observed in Experiment 1, we will assess the extent to which processing fluency and beliefs contribute to an animacy effect on JOLs. Specifically, we evaluated the contribution of processing fluency to the animacy effect either by measuring self-paced study time (Experiment 2a) or by presenting half the words in an easy or difficult font style (Experiment $2 b$ ). In Experiment 3, we examined whether participants had beliefs that animacy could influence their JOLs.

\section{Experiment 1}

Experiment 1 was an initial assessment of whether people used the animacy of words as a cue for JOLs. Participants were asked to learn 24 words (12 animate and 12 inanimate) and predict the likelihood of recalling each word on an upcoming test. Then after a 1-min distractor task consisting of arithmetic problems, participants were asked to take a recall 
test. There were two full study-test cycles in total. We predicted that participants would use animacy as a cue in making JOLs and would give higher JOLs to animate as compared to inanimate words.

\section{Method}

Participants Thirty-four undergraduates (20 females) with an average age of 20.37 years $(S D=1.21)$ participated in return for course credit or $¥ 10$.

Materials We chose 160 Chinese words ( 80 animate, 80 inanimate) from the Classification of Modern Chinese Dictionary (Dong 2007). We asked 24 independent raters to code the words in terms of their age of acquisition (7-point scale, $1=0-2$ years; 7 = age 13 and over), concreteness ( 5 -point scale, $1=$ very abstract; $5=$ very concrete $)$, familiarity (5-point scale, 1 = very unfamiliar with the meaning of the word; $5=$ very familiar with the meaning of the word $)$, and imagery $(1=$ very easy image; $5=$ very difficult image). Word frequency and stroke number were taken from Modern Chinese Frequency Dictionary (Wang 1986). After being matched along eight mnemonically relevant dimensions that affect Chinese words, we ultimately selected 24 words (12 animate and 12 inanimate, see Appendix A). For additional information about these words and dimensions, see Table 1. Four additional "buffer" words were chosen using the same procedure and matching process.

Design The experiment employed a $2 \times 2$ repeated-measures design with target word type (animate or inanimate) and study-test trial (1 or 2) manipulated as within-subjects factors. The proportion of correctly recalled items and JOLs were the dependent variables.

Procedure Participants were tested individually after giving their informed consent.

Participants were told that they were participating in a memory experiment, and they were asked to try to remember each word as it was presented. All 24 words were presented in random order to each participant. Each word appeared for $2 \mathrm{~s}$, with a 250-ms intertrial interval. At the beginning and end of the list, two buffer words were added (one of each type). These words were not scored in recall. After each item was presented, participants made a JOL on six discrete response options: $0 \%, 20 \%, 40 \%, 60 \%, 80 \%$, and $100 \%(0 \%=$ definitely will not remember the word $; 100 \%=$ definitely will remember the word). After all 28 items were presented, participants completed a 1-min distractor task consisting of arithmetic problems. Immediately after the distractor task, participants were asked to recall and write the words from the study phase, in any order, and were given 4 min to complete the task.
When participants had finished the recall period, they repeated the study, JOL, and recall procedures.

\section{Results and discussion}

The results of recall task were shown in Table 2. A strong recall advantage was present for animate items on each of the two trials. A $2 \times 2$ repeated-measures ANOVA conducted on recall performance (see Table 2) confirmed a significant main effect of word type, $F(1,33)=100.01, p<.001$, $\eta^{2}=.75$, and trial, $F(1,33)=34.80, p<.001, \eta^{2}=.51$, but no interaction, $F<1$.

More importantly, the results of the JOL task showed a strong JOL advantage for the animate items on each of the two trials (see Table 2). A $2 \times 2$ repeated-measures ANOVA confirmed a significant main effect of word type, $F(1,33)=$ 23.18, $p<.001, \eta^{2}=.41$, and trial, $F(1,33)=17.63, p<.001$, $\eta^{2}=.35$, but no interaction, $F<1$.

To examine the relative accuracy of JOLs, GoodmanKruskal gamma correlations were run to investigate the relationship between JOLs and recall performance (Nelson, 1984). JOL-recall gammas were calculated separately for animate and inanimate words. Two participants were excluded because they recalled all animate or inanimate words on Trial 2. The results showed that JOLrecall gammas reliably differed from zero for animate words $(G=0.17 ; S E=0.07), t(31)=2.59, p<.05, d=$ 0.46 , and inanimate words $(G=0.20 ; S E=0.08), t(31)=$ $2.65, p<.05, d=0.46$. A $2 \times 2$ repeated-measures ANOVA showed that JOL-recall gammas increased across trials, $F(1,31)=4.13, p=.051, \eta^{2}=.12$. The main effect of animacy and interaction were not significant, $F_{\mathrm{S}}<1$.

To measure whether people were reliant on animacy as a cue on Trial 1, but more reliant on mnemonic cues on Trial 2, we calculated the gamma correlation between animacy (i.e., 1 $=$ animate, $0=$ inanimate) and JOLs (see Zimmerman \& Kelley, 2010, for a similar analysis in the case of emotionality as a cue). A high JOL-animacy gamma would indicate that animacy is used as a cue for JOLs. A pairedsamples $t$ test on gamma correlations revealed no significant difference between trial $1(M=0.30, S D=0.36)$ and trial $2(M$ $=0.26, S D=0.38), t(34)<1$. This pattern indicates that animacy is a cue used to make JOLs on both trials.

The results of recall performance replicated the findings of previous studies (Bonin et al., 2014; Nairne et al., 2013; VanArsdall et al., 2013; VanArsdall et al., 2014), and expanded them to Chinese words. More critically, animacy was used as a cue for metamemory judgments, with participants assigning higher JOLs to animate than they did to inanimate words. In addition, as suggested by cue-utilization theory (Koriat, 1997), the impact of processing fluency (as a type of mnemonic cue) on JOLs should increase with study-test experience. However, we did not find a significant interaction 
Table 1 Characteristics of Control Variables in Experiments 1 and 2 for Animate and Inanimate Stimuli

\begin{tabular}{|c|c|c|c|c|}
\hline Dimension & Animate, $M(S D)$ & Inanimate, $M(S D)$ & $d f$ & $t$ \\
\hline Word frequency & $.0052^{1}(.0081)$ & $.0022(.0025)$ & 22 & $1.22, n . s$. \\
\hline Stroke number & $14.50^{2}(4.89)$ & $16.17(5.04)$ & 22 & $<1$ \\
\hline First stroke number & $7.58(3.00)$ & $8.67(4.48)$ & 22 & $<1$ \\
\hline Second stroke number & $6.92(4.27)$ & $7.50(3.06)$ & 22 & 1.04, n.s. \\
\hline Age of acquisition & $3.79(.30)$ & $3.83(.27)$ & 22 & $<1$ \\
\hline Concreteness & $4.27(.47)$ & $4.07(.38)$ & 22 & $1.11, n . s$. \\
\hline Familiarity & $4.37(.13)$ & $4.43(.13)$ & 22 & $-1.10, n . s$ \\
\hline Imagery & $1.62(.26)$ & $1.81(.33)$ & 22 & -1.59, n.s. \\
\hline
\end{tabular}

${ }^{1}$ The overall token is 0.0131 million (Wang 1986, Part 1). The average token of animate words is 68 , so the average frequency is 0.0052 (i.e., 68 occurrences in 0.0131 million)

${ }^{2}$ A stroke is defined as writing motion from pen down to pen up when one writes on a digitizer with a stylus pen (Liu, Cham, \& Chang, 1996), just as number of letters in English words. Moreover, the Chinese words (i.e., 狮子, lion) in our experiments are composed of two words (i.e., “狮” and “子””), so they need to be differentiated

between animacy type and study trial. This result might hint that processing fluency is not the driving force behind the animacy effect. We further explore the contribution of processing fluency in Experiment 2a and 2b.

\section{Experiment 2a}

Evidence from Experiment 1 suggests that animacy affects JOLs, as participants assigned higher JOLs to animate words than they did to inanimate words. This may have occurred because the animacy effect on JOLs is mediated by differential processing fluency. In Experiment 2a, we investigated the possibility that processing fluency, as measured by study time (e.g., Castel et al., 2007; Koriat, 2008; Koriat \& Ma'ayan, 2005; Koriat, Ma'ayan, \& Nussinson, 2006; Miele, Finn, \& Molden, 2011; Mueller et al., 2014; Undorf \& Erdfelder, 2015), mediates the relationship between animacy and JOLs. Study time is inversely related to JOLs (e.g., Castel et al., 2007; Koriat, 2008; Undorf \& Erdfelder, 2011), and hence is arguably a candidate for mediating the animacy effect on JOLs. For example, Undorf and Erdlelder (2015) examined whether differences in self-paced study time could account for why JOLs were higher for related pairs than for unrelated pairs. Consistent with their prediction, mean self-paced study time was lower for related pairs than for unrelated pairs.

Table 2 Mean Recall Performance and JOLs (SD) by Trial and Word Type in Experiment 1

\begin{tabular}{llllll}
\hline & \multicolumn{2}{l}{ Recall performance } & & JOLs & \\
\cline { 2 - 3 } \cline { 5 - 6 } & Trial 1 & Trial 2 & & Trial 1 & Trial 2 \\
\hline Animate & $0.47(0.17)$ & $0.66(0.21)$ & & $0.52(0.19)$ & $0.66(0.18)$ \\
Inanimate & $0.34(0.16)$ & $0.56(0.20)$ & & $0.45(0.17)$ & $0.59(0.18)$ \\
\hline
\end{tabular}

Furthermore, bootstrapped mediation analyses revealed that up to $15 \%$ of the relatedness effect was mediated by selfpaced study time. In the present case, if processing fluency was used as a cue for JOLs, then participants should allocate less study time to animate words than to inanimate words.

\section{Method}

Participants Thirty-eight undergraduates (26 females) with an average age of 21.16 years $(S D=1.53)$ participated in return for course credit or $¥ 10$.

Materials The materials were the same as those used in Experiment 1.

Design The only within-subjects variable was animacy (animate vs. inanimate).

Procedure The procedure was the same as that used in Experiment 1, except that the words were self-paced - participants were told to press the spacebar on the keyboard when they had finished studying the word. After a word was presented for study, a JOL rating was made for that word. After participants had studied and made JOLs for all the words, they completed a 3-min distractor task consisting of arithmetic problems. Last, participants completed a free-recall test for 4 min. Additionally, we only utilized one study-JOL-recall trial.

\section{Results and discussion}

More animate words were correctly recalled $(M=0.63, S D=$ $0.17)$ than were inanimate words $(M=0.53, S D=0.20), t(37)$ $=4.57, p<.001, d=.78$. With regard to memory predictions, participants provided higher JOLs for animate words $(M=0.58, S D=0.17)$ than they did for inanimate 
words $(M=0.52, S D=0.16), t(37)=4.35, p<.001, d=$ .81 . These results again suggest that animacy has a robust effect on memory and metamemory.

Median study times for animate and inanimate words were $4.18 \mathrm{~s}$ and $4.30 \mathrm{~s}$, respectively. A sign test revealed no reliable difference between animate and inanimate words, $p=.14$. In order to evaluate the hypothesis that processing fluency mediates the animacy effect on JOLs, we computed withinparticipant Pearson correlations (e.g., Mueller et al., 2015; Mueller et al., 2013) between each participant's JOLs and animacy, JOLs and self-paced study time, and JOLs and animacy, while controlling for self-paced study time. The results showed that the mean correlation between JOLs and animacy was $.16, t(37)=5.33, p<.001$, and -.05 between JOLs and self-paced study time, $t(37)=-1.38, p=.18$. Most importantly, after controlling for self-paced study time, the correlation between JOLs and animacy was .15, $t(37)=4.74$, $p<.001$, which did not differ from the zero-order correlation between animacy and JOLs, $t(37)<1$. Thus, study time did not mediate the relationship between animacy and JOLs.

As in Experiment 1, we report gamma correlations between recall and JOLs for each animacy condition. One participant was excluded because they gave all inanimate words a JOL of $80 \%$. The JOL-recall gammas reliably differed from zero for animate words $(G=0.45 ; S E=0.08), t(36)=5.57, p<.01, d=0.91$, and inanimate words $(G=0.19 ; S E=0.08), t(36)=2.31, p<.01, d=$ 0.37 . In addition, JOL-recall gammas for animate words differed between Experiment 1 (.17) and 2a (.45), t(67) $=2.64, p<.05$. However, to preview, this finding did not replicate in Experiment $2 \mathrm{~b}$ and will not be discussed further. A follow-up test showed that the resolution was lower overall for inanimate words compared to animate words, $t(36)=2.82, p<.01, d=0.47$.

\section{Experiment 2b}

Evidence from Experiment 2a suggests that processing fluency (i.e., as measured by self-paced study time) is not the factor mediating the animacy effect. However, there are other types of processing fluency that have been described in the literature (Alter \& Oppenheimer, 2009). For example, Rhodes and Castel (2008) found that disrupting processing fluency by presenting words in an alternating format diminished the font size effect, suggesting that processing fluency plays a role in the font size effect. Thus, in Experiment 2b, we used a font manipulation to alter the ease with which words could be read. Words presented in a difficult font style would disrupt participants' processing fluency (Alter \& Oppenheimer, 2009; Jia et al., 2016; Mueller et al., 2013; Rhodes \& Castel, 2008). If processing fluency plays a role in the animacy effect on JOLs, then this effect would decrease in magnitude or disappear when the words were presented in a difficult font style, compared with an easy font style.

\section{Method}

Participants Twenty-three undergraduates (15 females) with an average age of 23.2 years ( $S D=1.93$ years) were recruited to participate in the experiment. None of them had ever taken part in a similar experiment.

Design This experiment used a 2 (Animacy [animate or inanimate] $) \times 2$ (Font Style [easy or difficult]) within-subjects design. For the easy font style, words were printed in Imitation Song, a bold font, such as 机器 (machine). In the difficult font style, the words were printed in Teng cheung, a similar bold but also italicized font, such as姑 娘 (girl). The words printed in the easy font style were much more easier to $\operatorname{read}(M=1.05, S D=0.22)$ than those printed in the difficult font style $(M=2.95, S D=0.74), t(20)=-12.46, p<.001, d=$ 2.71 (see also Jia et al., 2016).

Materials The same materials were used as in Experiment 1. Each set of 12 animate words and 12 inanimate words were randomly divided into two subsets of six words, respectively. One subset was presented in an easy font and the other was presented in a difficult font. The four subsets were equated for word frequency, stroke number, age of acquisition, familiarity, concreteness and imagery $(p \mathrm{~s}>0.1)$. Four additional buffer words (two of each font styles) were always presented at the beginning and end of the list, respectively. They were excluded from all reported analyses.

Procedure The procedure was the same as Experiment 2a, except that each of the words was studied for $2 \mathrm{~s}$.

\section{Results and discussion}

Mean proportions of words correctly recalled were computed. A 2 (Animacy [animate or inanimate] $) \times 2$ (Font Style [easy or difficult]) ANOVA revealed a significant main effect for animacy, $F(1,22)=12.64, p<.01, \eta^{2}=.37$, indicating that participants' recall performance was higher for animate words $(M=.40, S E=.04)$ than for inanimate words $(M=.28, S E=$ $.04)$. No significant difference was found between recall performance for easy and difficult font style words, $F<1$. The interaction was not significant, $F(1,22)=4.07, p>.05$.

A 2 (Animacy [animate or inanimate]) $\times 2$ (Font Style [easy or difficult]) ANOVA on JOLs revealed a significant main effect for animacy, $F(1,22)=4.48, p<.05, \eta^{2}=.17$, indicating that participants gave higher JOLs for animate words $(M=.52, S E=.04)$ than they did for inanimate words $(M=.49, S E=.04)$. In addition, there was also a significant main effect of font style, $F(1,22)=4.29, p<.05, \eta^{2}=.16$, indicating that participants gave higher JOLs for words presented in an easy font $(M=.53, S E=.04)$ than they did for words presented in a difficult font style $(M=.48, S E=.04)$. 
However, the interaction between animacy and font style was not significant, $F<1$, suggesting that differences in processing fluency have no contribution to the animacy effect on JOLs.

The JOL-recall gammas reliably differed from zero for animate words $(G=0.22 ; S E=0.10), t(22)=2.12, p<.05, d=$ 0.45 , inanimate words $(G=0.25 ; S E=0.11), t(22)=2.17, p<$ $.05, d=0.44$, difficult font style words $(G=0.28 ; S E=0.10)$, $t(22)=2.76, p<.05, d=0.58$, and easy font style words $(G=$ $0.21 ; S E=0.09), t(22)=2.20, p<.05, d=0.47$. A $2 \times 2$ repeated measures ANOVA did not show any effects, $p \mathrm{~s}>.62$.

\section{Experiment 3}

Evidence from Experiment $2 \mathrm{a}$ and $2 \mathrm{~b}$ suggests that processing fluency plays a minimal role in the animacy effect on JOLs. Accordingly, in Experiment 3, we changed our focus to examine whether people have a belief about animacy. We designed a questionnaire to evaluate this possibility. This is a standard method for attempting to isolate theory-based influences on metamemory (e.g., Koriat et al., 2004; Kornell et al., 2011; Mueller et al., 2015; Mueller et al., 2014; Susser \& Mulligan, 2014). For example, Mueller et al. (2013) had participants read a description of a hypothetical experiment wherein students studied both related and unrelated pairs and were tested on them. After reading about this experiment, participants estimated how many related and unrelated pairs they expected that the students would recall. The results showed that participants' estimates were higher for related than for unrelated pairs. These data indicate that people have a belief about the relative memorability of related and unrelated pairs. Using the same method, we had participants read a description of an experiment and predicted recall performance for animate and inanimate words without actually having the experience that participants in the actual experiment had. In addition, we asked participants to provide an explanation for why they thought animate [inanimate] words would be remembered better than inanimate [animate] words (depending on the estimates made by a given participant).

\section{Method}

Participants Thirty-one undergraduates (20 females) with an average age of 22.03 years $(S D=1.30)$ participated in return for course credit or $¥ 10$.

Materials, design, and procedure Participants read the following description of an experiment:

In a previous experiment that we conducted, participants were presented with 24 Chinese words on the computer screen, one after the other. Critically, these words consisted of two types: half of the words were animate and the other half were inanimate. Each of the words was randomly presented for $2 \mathrm{~s}$. Participants' task was to study these words so that they would remember as many words as possible on a memory test. Participants then solved math problems in their heads for 2 min. Finally, they were asked to write down as many words as they could remember from the first part of the experiment. They could write the words in any order that they wanted.

On the questionnaire, an example of the animate and inanimate words was provided.

Furthermore, the order of the description of animacy words and the order of estimates for each animacy word were counterbalanced across participants. After reading the scenario, participants estimated the number of words (out of 12) of each type (animate and inanimate) that the participants in the experiment would recall.

\section{Results and discussion}

A paired-samples $t$ test on predictions revealed that participants gave higher estimates for animate words $(M=0.59$, $S D=0.17)$ than they did for inanimate words $(M=0.50, S D$ $=0.15), t(30)=4.26, p<.001, d=.80$. Twenty-five participants estimated higher recall for animate words. Their explanations were as follows: (a) animate words are easier to contact with regard to actual life as compared to inanimate words $(n=14)$; (b) animate words are easier to remember than are inanimate words $(n=19)$; (c) animate words are more vivid than are inanimate words $(n=17)$; (d) it is easier to produce mental pictures for animate words than it is for inanimate words $(n=8)$; and (e) animate words are in the same category as participants $(n=18)$. Four indicated that inanimate words would be better remembered because inanimate words are commonly used in their daily communication and life. Two participants responded that the two types would be remembered equally. These results suggest that people have some beliefs about the effect of animacy on memory, indicating that the results of Experiments 1, 2a, and 2b were driven by such beliefs. Furthermore, peoples' beliefs as reflected in this questionnaire are more in line with actual memory performance in Experiments 1, 2a, and 2b. Therefore, it is possible that peoples' beliefs override processing fluency.

\section{General discussion}

The current study investigated the role of the animacy of words in memory monitoring and the degree to which processing fluency and beliefs contribute to the animacy effect on JOLs. In Experiments 1, participants predicted that animate words would be more memorable as compared to inanimate words. In Experiment 2a, processing fluency (as measured by self- 
paced study time) did not statistically mediate any of the variability between animacy cue and JOLs. In Experiment 2b, the size of animacy effect did not decrease or disappear even when presenting words in a difficult font style. In the questionnairebased Experiment 3, participants estimated higher recall for animate than for inanimate words. These results suggest that animacy is a cue that people rely on when monitoring their learning, and furthermore, that belief as opposed to processing fluency contributes substantially to the animacy effect on JOLs.

It is unsurprising that participants attended to animacy in their JOLs. With the words being matched on several dimensions (e.g., concreteness, familiarity, imagery), the animacy of words likely became a salient cue for JOLs. This conclusion is consistent with Koriat's (1997) cue-utilization framework, which suggests that JOLs may be based on intrinsic cues defined as information inherent to the items being studied. For instances, Zimmerman and Kelley (2010) found that emotionality (i.e., one type of intrinsic cue) is a cue that people rely on when monitoring their learning of emotional and neutral materials. In addition, the outcomes from Experiment 1 showed that multiple cues - in this case, animacy and number of presentations - can simultaneously inform JOLs. This is consistent with the idea of cue-weighting (Koriat, 1997), in which multiple factors inform JOLs. The finding that these cues operated independently, reflected by the lack of an interaction, is consistent with previous researches (e.g., Koriat et al., 2006; Soderstrom \& McCabe, 2011).

The outcomes from Experiment 2a were surprising, as they did not provide support for the processing fluency hypothesis. The animacy of words did not lead to more fluent processing of items, as measured by self-paced study time. In line with the processing fluency hypothesis, Koriat et al. (2006) repeatedly found negative correlations between JOLs and self-paced study time. However, we found no expected negative relationship between study time and JOLs, self-paced study time also failed to mediate the relationship between animacy cue and JOLs. One possible explanation is that the animacy cue overshadowed the use of study time as a cue. Consistent with this possibility, Evans and Benjamin (2011) review evidence that "fluency cues appear to be more important under conditions in which other bases for the recognition decisions ... were reduced" (pp. 20-21). That is, when other cues are available for participants to retrieve an item, fluency no longer influences their JOLs (Mueller et al., 2014). For example, Mueller et al. (2015) found that the correlation between study time and JOLs was -.02 and nonsignificant (see also Jia et al., 2016, mean $r=$ .04 and nonsignificant; Mueller et al., 2014, mean $r=.06$ and nonsignificant), suggesting that the intrinsic cue (i.e., word frequency, identical pairs, and font size) overshadows the use of study time as a cue. Another possible explanation is that the nonsignificant relationship between study time and JOLs may be mediated by an analytic mode of processing. For example, Koriat and Ackerman (2010) found that when participants made JOLs for themselves, study time was negatively related to JOLs. However, when participants made JOLs for another learner (while observing the variability in study times for that learner) prior to for themselves, study time and JOLs were not related (see also, Undorf \& Erdfelder, 2011, 2013). According to Koriat and Ackerman (2010), recall predictions for others could presumably use an analytic process, that is, deliberate use of one's theories and beliefs about memory. Thus, these results suggest that the non-significant relationship between study time and JOLs (for those who made JOLs for others) may be mediated by an analytic mode of processing.

Of course, other measures and manipulations of processing fluency should be considered (Alter \& Oppenheimer, 2009), such as the numbers of trials needed for correct recall (Koriat, 2008; Undorf \& Erdfelder, 2015), the speeds with which participants form an interactive image of the cue and target of paired associates (Hertzog et al., 2003), response times in a lexical decision task (Mueller et al., 2014), and the subjective experience of ease with which the stimulus is processed (Jia et al., 2016). In our Experiment 2b, we used a font style manipulation to alter the ease with which words could be read. The results showed that differences in processing fluency contribute minimally to the animacy effect on JOLs. However, we still should be cautious about this conclusion because we may not be able to entirely rule out the possibility of processing fluency mediating the animacy effect on JOLs. Further research is needed to use other measures of processing fluency, for instance, lexical decision response times (e.g., Mueller et al., 2013), to further explore the role of processing fluency in the effect of animacy on JOLs.

Evidence from Experiment 3 suggests that beliefs mediate the animacy effect on JOLs. One possibility is that people hold some beliefs about the influence of animacy on memory prior to participating in these experiments. As suggested by New et al. (2007), remembering which humans were family, friends, adversaries, or potential mates is critical for survival and reproduction. Information about nonhuman animals was also of critical importance to our foraging ancestors (New et al., 2007). These beliefs may result from everyday experiences with animacy or from formal learning. Another possibility is that beliefs may be developed online as participants consider how animacy could potentially influence memory. For instance, Tauber and Rhodes (2010, Experiment 3) found that during the first study-test of face-noun pairs (e.g., facename or occupation) trial, the cue of a name or occupation did not influence JOLs, but on the second study-test trial with new pairs, participants gave higher JOLs for face-occupation pairs than they did for face-name pairs. These results suggest that participants learned from task experience during the first trial (e.g., occupations were better remembered than were names), and this new experience influenced JOLs on the second trial. In addition, as suggested by AP theory of JOLs (Dunlosky, Mueller, \& Tauber, 2014; Mueller et al., 2015), an explicit instruction to focus on accurately predicting memory may 
trigger an analytic problem-solving mode. That is, in order to reduce uncertainty about the memory prediction, participants may seek available cues to guide their predictions (Dunlosky et al., 2014; Mueller et al., 2015). For example, in the case of both the font size (Mueller et al., 2014) and identical effects (Mueller et al., 2015) on JOLs, participants may have developed an explanation by reading the experimental instructions or searching for cues (i.e., font size, identical) to reduce their uncertainty about predictions. Overall, people's beliefs about a cue may arise from a variety of sources (Mueller et al., 2015), and further studies should focus on what these beliefs are and when they form (Dunlosky et al., 2014).

In sum, the current results are important for at least two primary reasons. First, they extend the generality of the animacy advantage to retention of a new set of stimuliChinese words. This finding provides further convincing evidence for the functional view of human memory. Given that our ancestors were primarily concerned with survival, the ability to rapidly detect and remember animates in the visual field and determine whether they were potential predators or prey would seem to be a highly advantageous skill. As argued by Nairne and coworkers (e.g., Nairne \& Pandeirada, 2008, 2010; Nairne et al., 2009), the human memory system is based or tuned to remember fitness-relevant information, based essentially on findings obtained using survival processing scenarios. For instance, Nairne et al. (2008) found that processing stimuli in terms of fitness yields better memory performance than does processing them in terms of other dimensions corresponding to well-known deep encoding conditions. In most studies investigating the survival processing advantage, the survival effect has mostly been obtained by varying the encoding contexts. The present research further supports this view by using words more-or-less related to adaptive problems. Second, the current results suggest that animacy is an intrinsic cue that people rely on when monitoring their learning, which is consistent with the cue-utilization framework of JOLs (Koriat, 1997). More importantly, Experiments 2a, 2b, and 3 showed that the animacy effect on JOLs is mediated by beliefs, not processing fluency, which is incompatible with the cue-utilization framework. According to the AP theory of JOLs (Mueller et al., 2015), if participants cannot develop some plausible explanations for why and how a cue may influence memory predictions, then the differences in processing fluency will affect JOLs. Therefore, we cannot entirely rule out the contribution of processing fluency. Given that fluency is an important theoretical construct in many perspectives on judgments and decision making (Unkelbach \& Greifeneder, 2013), further research should seek to illustrate the contribution of processing fluency to the effect of animacy on JOLs by using different measures and manipulations.

Acknowledgments This research was supported by the National Natural Science Foundation of China (31170999).

\section{Appendix}

Table 3 Chinese words and its English translations used in Experiment 1 and 2

\begin{tabular}{lllll}
\hline Animate & & & Inanimate & \\
\cline { 1 - 2 } \cline { 5 - 5 } $\begin{array}{llll}\text { Chinese } \\
\text { words }\end{array}$ & $\begin{array}{l}\text { English } \\
\text { translations }\end{array}$ & & $\begin{array}{l}\text { Chinese } \\
\text { words }\end{array}$ & $\begin{array}{l}\text { English } \\
\text { translations }\end{array}$ \\
\hline 狮子 & lion & & 门窝 & curtain \\
乌鸦 & crow & & 笼子 & coop \\
松鼠 & squirrel & & 塑料 & plastic \\
飞蛾 & moth & & 煤炭 & coal \\
护士 & nurse & & 木材 & wood \\
熊猫 & panda & & 暖壸 & thermos \\
妇女 & woman & & 公园 & park \\
知了 & cicada & 衣领 & collar \\
婴儿 & baby & 茶几 & teapoy \\
姑娘 & girl & 轮船 & steamer \\
金鱼 & goldfish & 餐厅 & restaurant \\
司机 & driver & 书桌 & desk \\
\hline
\end{tabular}

\section{References}

Alban, M. W., \& Kelley, C. M. (2013). Embodiment meets metamemory: Weight as a cue for metacognitive judgments. Journal of Experimental Psychology: Learning, Memory \& Cognition, 39(5), 1628-1634. doi:10.1037/a0032420

Alter, A. L., \& Oppenheimer, D. M. (2009). Uniting the tribes of fluency to form a metacognitive nation. Personality \& Social Psychology Review, 13(3), 219-235. doi:10.1177/1088868309341564

Begg, I., Vinski, E., Frankovich, L., \& Holgate, B. (1991). Generating makes words memorable, but so does effective reading. Memory \& Cognition, 19(5), 487-497. doi:10.3758/BF03199571

Besken, M., \& Mulligan, N. W. (2013). Easily perceived, easily remembered? Perceptual interference produces a double dissociation between metamemory and memory performance. Memory \& Cognition, 41(6), 897-903. doi:10.3758/s13421-013-0307-8

Bonin, P., Gelin, M., \& Bugaiska, A. (2014). Animates are better remembered than inanimates: Further evidence from word and picture stimuli. Memory \& Cognition, 42(3), 370-382. doi:10.3758/s13421013-0368-8

Caramazza, A., \& Mahon, B. Z. (2003). The organization of conceptual knowledge: The evidence from category-specific semantic deficits. Trends in Cognitive Sciences, 7(8), 354-361. doi:10.1016/S13646613(03)00159-1

Castel, A. D., McCabe, D. P., \& Roediger, H. L. (2007). Illusions of competence and overestimation of associative memory for identical items: Evidence from judgments of learning. Psychonomic Bulletin \& Review, 14(1), 107-111. doi:10.3758/BF03194036

Dong, D. (Eds.). (2007). Classification of modern Chinese dictionary (in Chinese). Shanghai, China: Shanghai Lexicographic Publishing House. 
Dunlosky, J., Mueller, M., \& Tauber, S. K. (2014). The contribution of processing fluency (and beliefs) to people's judgments of learning. In D. S. Lindsay, C. M. Kelley, A. P. Yonelinas, \& H. L. Roediger III (Eds.), Remembering: Attributions, processes, and control in human memory: Papers in honour of Larry L. Jacoby (pp. 46-64). New York: Psychology Press.

Evans, K. M., \& Benjamin, A. S. (2011). Fluency and familiarity: How memory for perceptual detail influences the remembering events. In P. A. Higham \& J. P. Leboe (Eds.), Constructions of remembering as metacognition (pp. 17-27). Basingstoke: Palgrave Macmillan.

Hertzog, C., Dunlosky, J., Robinson, A. E., \& Kidder, D. P. (2003). Encoding fluency is a cue used for judgments about learning. Journal of Experimental Psychology: Learning, Memory \& Cognition, 29(1), 22-34. doi:10.1037/0278-7393.29.1.22

Jia, X., Li, P., Li, X., Zhang, Y., Cao, W., Cao, L., \& Li, W. (2016). The effect of word frequency on judgments of learning: Contributions of beliefs and processing fluency. Frontiers in Psychology, 6, 1995. doi:10.3389/fpsyg.2015.01995

Koriat, A. (1997). Monitoring one's own knowledge during study: A cueutilization approach to judgments of learning. Journal of Experimental Psychology: General, 126(4), 349-370. doi:10. 1037/0096-3445.126.4.349

Koriat, A. (2008). Easy comes, easy goes? The link between learning and remembering and its exploitation in metacognition. Memory \& Cognition, 36(2), 416-428. doi:10.3758/MC.36.2.416

Koriat, A., \& Ackerman, R. (2010). Metacognition and mindreading: Judgments of learning for self and other during self-paced study. Consciousness and Cognition, 19(1), 251-264. doi:10.1016/j. concog.2009.12.010

Koriat, A., \& Ma'ayan, H. (2005). The effects of encoding fluency and retrieval fluency on judgments of learning. Journal of Memory and Language, 52(4), 478-492. doi:10.1016/j.jml.2005.01.001

Koriat, A., Bjork, R. A., Sheffer, L., \& Bar, S. K. (2004). Predicting one's own forgetting: The role of experience-based and theory-based processes. Journal of Experimental Psychology: General, 133(4), 643656. doi:10.1037/0096-3445.133.4.643

Koriat, A., Ma'ayan, H., \& Nussinson, R. (2006). The intricate relationships between monitoring and control in metacognition: Lessons for the cause-and-effect relation between subjective experience and behavior. Journal of Experimental Psychology: General, 135(1), 3669. doi:10.1037/0096-3445.135.1.36

Kornell, N., Rhodes, M. G., Castel, A. D., \& Tauber, S. K. (2011). The ease-of-processing heuristic and the stability bias: Dissociating memory, memory beliefs, and memory judgments. Psychological Science, 22(6), 787-794. doi:10.1177/0956797611407929

Li, W., Zhang, Y., Li, F., Li, X., Li, P., Jia, X., . . Ji, H. (2015). Out of lust or jealousy: The effects of mate-related motives on study-time allocation to faces varying in attractiveness. PLOS ONE, 10(6), e0132207. doi:10.1371/journal.pone.0132207

Liu, J., Cham, W. K., Chang, M. M. Y. (1996). Stroke order and stroke number free on-line Chinese character recognition using attributed relational graph matching. In R. Bolle \& E. Dickmanns (Eds.), Proceedings of the 13th International Conference on Pattern Recognition, Track C: Applications and Robotic Systems (pp.259263). Los Alamitos, California: IEEE Computer Society Press.

Lowder, M. W., \& Gordon, P. C. (2015). Natural forces as agents: Reconceptualizing the animate-inanimate distinction. Cognition, 136, 85-90. doi:10.1016/j.cognition.2014.11.021

Matvey, G., Dunlosky, J., \& Guttentag, R. (2001). Fluency of retrieval at study affects judgments of learning (JOLs): An analytic or nonanalytic. Memory \& Cognition, 29(2), 222-233. doi:10.3758/ BF03194916

Metcalfe, J., \& Jacobs, W. J. (2010). People's study time allocation and its relation to animal foraging. Behavioural Processes, 83(2), 213-221. doi:10.1016/j.beproc.2009.12.011
Miele, D. B., Finn, B., \& Molden, D. C. (2011). Does easily learned mean easily remembered? It depends on your beliefs about intelligence. Psychological Science, 22(3), 320-324. doi:10.1177/ 0956797610397954

Mueller, M. L., Tauber, S., \& Dunlosky, J. (2013). Contributions of beliefs and processing fluency to the effect of relatedness on judgments of learning. Psychonomic Bulletin \& Review, 20(2), 378-384. doi: 10.3758/s13423-012-0343-6

Mueller, M. L., Dunlosky, J., Tauber, S. K., \& Rhodes, M. G. (2014). The font-size effect on judgments of learning: Does it exemplify fluency effects or reflect people's beliefs about memory? Journal of Memory and Language, 70, 1-12. doi:10.1016/j.jml.2013.09.007

Mueller, M. L., Dunlosky, J., \& Tauber, S. K. (2015). The effect of identical word pairs on people's metamemory judgments: What are the contributions of processing fluency and beliefs about memory? The Quarterly Journal of Experimental Psychology, 1-19. doi: 10.1080/17470218.2015.1058404

Nairne, J. S., \& Pandeirada, J. N. S. (2008). Adaptive memory: Is survival processing special? Journal of Memory and Language, 59(3), 377385. doi:10.1016/j.jml.2008.06.001

Nairne, J. S., \& Pandeirada, J. N. S. (2010). Adaptive memory: Ancestral priorities and the mnemonic value of survival processing. Cognitive Psychology, 61(1), 1-22. doi:10.1016/j.cogpsych.2010.01.005

Nairne, J. S., Thompson, S. R., \& Pandeirada, J. N. S. (2007). Adaptive memory: Survival processing enhances retention. Journal of Experimental Psychology: Learning, Memory, and Cognition, 33(2), 263-273. doi:10.1037/0278-7393.33.2.263

Nairne, J. S., Pandeirada, J. N. S., \& Thompson, S. R. (2008). Adaptive memory: The comparative value of survival processing. Psychological Science, 19(2), 176-180. doi:10.1111/j.1467-9280. 2008.02064.x

Nairne, J. S., Pandeirada, J. N. S., Gregory, K. J., \& VanArsdall, J. E. (2009). Adaptive memory: Fitness relevance and the hunter-gatherer mind. Psychological Science, 20(6), 740-746. doi:10.1111/j.14679280.2009.02356.x

Nairne, J. S., VanArsdall, J. E., Pandeirada, J. S., Cogdill, M., \& LeBreton, J. M. (2013). Adaptive memory: The mnemonic value of animacy. Psychological Science, 24(10), 2099-2105. doi:10. $1177 / 0956797613480803$

Nelson, T. O. (1984). A comparison of current measures of the accuracy of feeling-of-knowing predictions. Psychological Bulletin, 95, 109-133.

New, J., Cosmides, L., \& Tooby, J. (2007). Category-specific attention for animals reflects ancestral priorities, not expertise. Proceedings of the National Academy of Sciences of the United States of America, 104(42), 16598-16603. doi:10.1073/pnas.0703913104

Palmore, C., Garcia, A., Bacon, L., Johnson, C., \& Kelemen, W. (2012). Congruity influences memory and judgments of learning during survival processing. Psychonomic Bulletin \& Review, 19(1), 119125. doi:10.3758/s13423-011-0186-6

Pratt, J., Radulescu, P. V., Guo, R. M., \& Abrams, R. A. (2010). It's alive! Animate motion captures visual attention. Psychological Science, 21(11), 1724-1730. doi:10.1177/0956797610387440

Rhodes, M. G., \& Castel, A. D. (2008). Memory predictions are influenced by perceptual information: Evidence for metacognitive illusions. Journal of Experimental Psychology: General, 137(4), 615625. doi:10.1037/a0013684

Soderstrom, N. C., \& McCabe, D. P. (2011). The interplay between value and relatedness as bases for metacognitive monitoring and control: Evidence for agenda-based monitoring. Journal of Experimental Psychology: Learning, Memory, and Cognition, 37(5), 12361242. doi: $10.1037 / \mathrm{a} 0023548$

Susser, J. A., \& Mulligan, N. W. (2014). The effect of motoric fluency on metamemory. Psychonomic Bulletin \& Review, 1-6. doi:10.3758/ s13423-014-0768-1

Szewczyk, J. M., \& Schriefers, H. (2011). Is animacy special?: ERP correlates of semantic violations and animacy violations in sentence 
processing. Brain Research, 1368, 208-221. doi:10.1016/j.brainres. 2010.10.070

Tauber, S. K., \& Rhodes, M. G. (2010). Metacognitive errors contribute to the difficulty in remembering proper names. Memory, $18(5), 522$ 532. doi:10.1080/09658211.2010.481818

Undorf, M., \& Erdfelder, E. (2011). Judgments of learning reflect encoding fluency: Conclusive evidence for the ease-of-processing hypothesis. Journal of Experimental Psychology: Learning, Memory, and Cognition, 37(5), 1264-1269. doi:10.1037/a0023719

Undorf, M., \& Erdfelder, E. (2013). Separation of encoding fluency and item difficulty effects on judgements of learning. The Quarterly Journal of Experimental Psychology, 66(10), 2060-2072. doi:10. 1080/17470218.2013.777751

Undorf, M., \& Erdfelder, E. (2015). The relatedness effect on judgments of learning: A closer look at the contribution of processing fluency. Memory \& Cognition, 43(4), 647-658. doi:10. 3758/s13421-014-0479-x
Unkelbach, C., \& Greifeneder. (2013). The experience of thinking: How the fluency of mental processes influences cognition and behavior. New York: Psychology Press.

VanArsdall, J. E., Nairne, J. S., Pandeirada, J. N., \& Blunt, J. R. (2013). Adaptive memory: Animacy processing produces mnemonic advantages. Experimental Psychology, 60(3), 172-178. doi:10.1027/ 1618-3169/a000186

VanArsdall, J. E., Nairne, J. S., Pandeirada, J. N. S., \& Cogdill, M. (2014). Adaptive memory: Animacy effects persist in pairedassociate learning. Memory, 1-7. doi:10.1080/09658211.2014. 916304

Wang, H. (Eds.). (1986). Modern Chinese frequency dictionary (in Chinese). Beijing, China: Beijing Language College Press.

Zimmerman, C. A., \& Kelley, C. M. (2010). "I'll remember this!" Effects of emotionality on memory predictions versus memory performance. Journal of Memory and Language, 62(3), 240-253. doi: 10.1016/j.jml.2009.11.004 